\title{
Distributed Energy Transaction Model Based on the Alliance Blockchain in Case of China
}

\author{
Yongxiu He ${ }^{1,2}$, Wei Xiong ${ }^{1,3}$, Bin-you Yang ${ }^{3}$, Rui Zhang ${ }^{3}$, \\ Ming-li Cui ${ }^{4}$, Tian-tian Feng ${ }^{5,6,7, *}$ and Yi-er Sun ${ }^{8, *}$ \\ ${ }^{1}$ School of Economics and Management, North China Electric Power University, \\ Changping Beijing, China \\ ${ }^{2}$ Beijing Key Laboratory of New Energy and Low-Carbon Development \\ (North China Electric Power University), Changping Beijing, China \\ ${ }^{3}$ State Grid Hunan Electric Power Company limited, Changde Power Supply \\ Company, Changde, Hunan, China \\ ${ }^{4}$ School of Economics and Business Administration, Heilongjiang University, \\ Harbin, China \\ ${ }^{5}$ School of Economics and Management, China University of Geosciences, \\ Beijing, China \\ ${ }^{6}$ Key Laboratory of Carrying Capacity Assessment for Resource and Environment, \\ Ministry of Land and Resources, Beijing, China \\ ${ }^{7}$ Key Laboratory of Strategic Studies, Ministry of Land and Resources, \\ Beijing, China \\ ${ }^{8}$ School of Engineering, The Hong Kong University of Science and Technology, \\ Hong Kong, China \\ E-mail:fengtiantian89@163.com; sunyier98sino@163.com \\ * Corresponding Author
}

Received 13 November 2020; Accepted 08 December 2020;

Publication 09 March 2021

\section{Abstract}

Distributed energy, mainly composed of new energy, plays an important role in promoting the development of new energy. At present, the development

Journal of Web Engineering, Vol. 20_2, 359-386.

doi: 10.13052/jwe1540-9589.2026

(C) 2021 River Publishers 
of distributed energy is greatly hindered by imperfect trading platform and unstable output of new energy. Blockchain is decentralized, autonomous and requires collaborative management. Its own technical characteristics have the inherent advantages of reconstructing the energy system. The alliance chain in the blockchain is more suitable for building a distributed energy trading platform. The paper constructs a distributed energy transaction model based on alliance blockchain, studies the integration mode of blockchain and distributed energy transaction, and explores the application of blockchain in distributed transaction. The paper provides a new idea for optimizing and reconstructing the traditional distributed energy trading platform, and providing decision support for promoting distributed energy trading.

Keywords: Blockchain, distributed energy trading, energy Internet.

\section{Introduction}

Distributed energy is mainly composed of new energy and plays an increasingly important role in the energy trading market [1]. Distributed energy can promote the development of new energy and carbon emission reduction. However, the development of distributed energy is hindered by the imperfect trading platform, the instability of new energy and the impact of new energy on the power grid [2]. The paper constructs a distributed energy trading model based on alliance chain, aiming to promote distributed energy trading and optimize energy system by building a more optimized trading platform. The future energy system will support the access of large-scale distributed generation and energy storage system based on Internet technology, which can realize energy sharing in a wide area sense [3]. Accelerating energy flow through energy conversion equipment can significantly improve energy efficiency [4]. In the future, China's electricity market tends to be more diversified, convenient, and flexible [5, 6].

So far, many experts and scholars have studied blockchain and its application. Blockchain has four main characteristics: decentralization, transparency, contract execution automation and traceability [7]. Its core technologies mainly include distributed storage technology, cryptography, consensus mechanism and smart contract [8]. The hierarchical structure of blockchain is shown in Figure 1. The three most significant advantages of blockchain for data processing and analysis are: realizing automatic operation and transaction, reducing transaction and regulatory costs, and ensuring information 


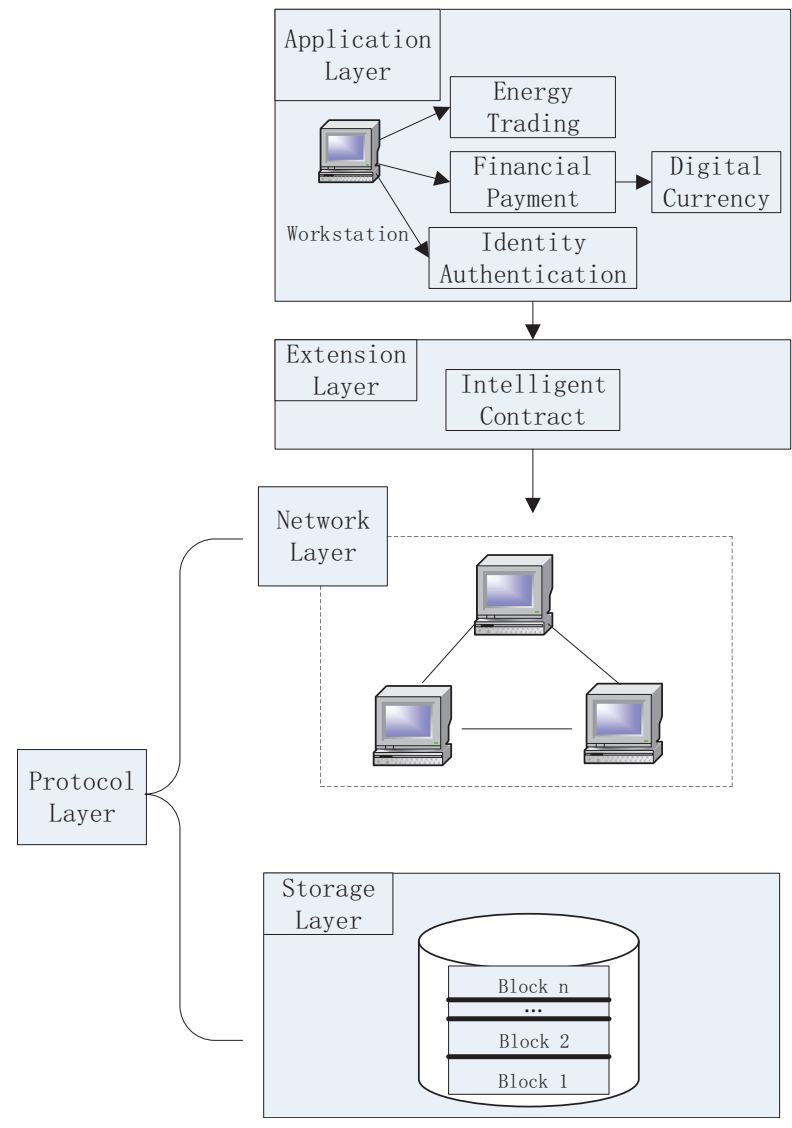

Figure 1 Hierarchical architecture of the block chain.

security. Literature [9] proposes the framework of blockchain technology and its typical application in energy Internet. Literature [10] and [11] design the settlement smart contract of electricity market transaction, analyse the key technical difficulties, and give the solutions. In Literature [12], an EV charging transaction model based on alliance chain is proposed, and the feasibility of the above method is verified by taking the actual distribution scenario of charging stations in some regions of Tianjin as an example. In Literature [13] and [14], the economic dispatch model of micro grid based on blockchain is studied, and the decentralized economic dispatch of micro grid is realized by using blockchain. In Literature [15], a multi 


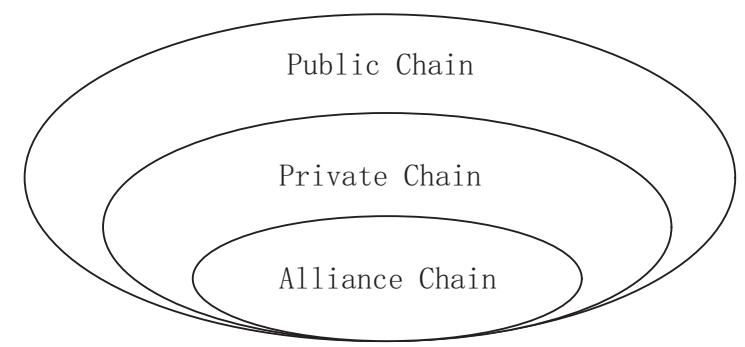

Figure 2 The inclusion relationships of three blockchains.

energy complementary security transaction model of heterogeneous energy blockchain is proposed to solve the problem of distributed energy multi energy complementary and integrated transaction in the energy transaction system.

Blockchain can be divided into public chain, private chain, alliance chain, etc., whose application scenarios are various due to different attributes. Figure 2 shows the inclusion relationship of the three blockchains.

The public chain is open to all entity users, nodes can be joined at will, and transactions cannot be tampered with [16]. It is important to build consensus and form a decentralized energy trading mechanism in the unfamiliar network environment. literature [17] suggests that the public chain is of great application value, and has its own unique characteristics in terms of technology innovation direction, technology application form, and technology innovation mode. In literature [24], it is proposed that the public chain is open to the public, and users can freely access the network without registration or authorization. In literature [18], a distributed energy Internet system based on the public chain is proposed, which can support multiuser and distributed energy access, and ensure the automation of contract execution and the transparency, irreversibility, and traceability of transactions on the chain. In literature [19] presents a public blockchain-based solution for the digitization of land ownership in Bangladesh, which embodies the characteristics of public chain data synchronization and transparency, easy access, and immutable record management.

A private chain is a closed blockchain that is open to a single individual or entity, with only private parties involved in bookkeeping, maintenance, and governance, and does not require the issuance of passes to incentivize nodes. Private chains are characterized by fast transaction speed, low transaction costs, and high security. literature [20], it is proposed that a healthcare security network access control system based on Ethereum private chain has 
stronger data security and system stability. In literature [21], it is proposed that since network security in the power and energy sector is directly related to power supply and power security, network security and privacy security in the IoT environment should not be underestimated.

An alliance chain is a chain of members and a limited number of third parties for a group, which internally designates several pre-selected nodes as bookkeepers, and the generation of each block is determined jointly by all the pre-selected nodes. In layman's terms, it is a semi-public blockchain, where only certain members can participate in bookkeeping privileges and maintenance governance, and can choose whether to issue a pass or not. In terms of security, an alliance chain is more secure than a public chain because it pays more attention to privacy and security, and in terms of trustworthiness, it is more trustworthy than a private chain because industries can monitor each other. In terms of cost, transactions in the alliance chain only need to be verified by a few trusted nodes with high arithmetic power, and there is no need for network-wide validation, so the transaction costs are lower. Literature [22], it is proposed that the use of Alliance blockchain technology can provide new ideas and methods for the copyright department to confirm the copyright of works and trace the source of piracy, which reflects the abovementioned characteristics of the Alliance chain such as high controllability and non-disclosure of data by default. In literature [23], it is pointed out that the microgrid based on the alliance chain has the advantages of high efficiency and easy supervision. In literature [24], it is proposed that alliance chains can be open to the public according to application scenarios, and that their networks are jointly maintained by member institutions and nodes are accessed through the gateway nodes of the member institutions. In literature [25], a comparative analysis between the unquoted electricity trading mechanism of the alliance chain and existing blockchain electricity trading schemes show that the former scheme benefits both buyers and sellers, with lower economic costs and higher consensus efficiency. The literature [26] points out that the limited design of the alliance blockchain and the study of provincial-scale partial trading scenarios are used to explore the ways of faster application of blockchain technology for early application in energy trading. In literature [27], it is pointed out that blockchain technology plays an important role in the construction of energy internet and power system reform based on the current development status of blockchain technology in energy internet, the improvement of blockchain technology, and the trend of energy internet construction in the five fields of energy supply, transmission, distribution, consumption, and trading. In literature [28], a new electric 
vehicle charging system is proposed, in which billing information is managed and recorded because of the alliance blockchain, which is tamper-resistant and polycentric and provides convenience for energy trading and cooperation. The literature [29] proposed a smart meter privacy protection scheme for distributed smart home environment based on alliance blockchain, using blockchain alliance distributed storage of user data, solving the problem of single point of failure and data tampering, the scheme ensures user privacy, and has confidentiality and non-falsifiability. The literature [30] proposes that the integration of blockchain technology with the field of renewable energy is a new cross-cutting research area of increasing interest, with blockchain fuelling renewable energy sources and powering energy sustainability. The literature [31] proposes a blockchain energy smart trading strategy to secure the transmission of energy and information flows between users, which increases the share of clean energy in the electricity market and ensures the stability of the energy trading market. The literature [32] analysed the competitiveness of distributed energy in China using the five forces model, and analysed the joint development model of blockchain technology + distributed energy using the SWOT model, which revealed the feasibility of applying blockchain technology to distributed energy systems and provided a theoretical basis for blockchain technology to improve the competitiveness of distributed energy. Literature [33] points out that alliance chain is more suitable for energy trading than public chain, and designs a decentralized energy trading system based on alliance chain. In Literature [34], basing on alliance chain's identity authentication protocol of the micro grid, the blockchain is applied to micro grids' identity authentication; it is explained that the scheme can solve the identity authentication problem safely and reliably, and can make the performance consumption of micro grid nodes to the minimum through scheme comparison, performance analysis, security certification and analysis.

Based on the research results of experts and scholars, this paper constructs a distributed energy trading model based on alliance blockchain, studies the integration mode of blockchain and distributed energy trading, analyses the application of blockchain in distributed trading, and provides new ideas for optimizing and reconstructing distributed energy trading platform to promote the development of distributed energy. To build the integration mode of blockchain and energy Internet, it can start with distributed energy transactions. The paper can provide a theoretical and policy support for the construction of energy Internet, and provide ideas for the integration of blockchain and energy Internet. 


\section{Distributed Energy Transaction Model Based on Alliance Chain}

The distributed energy trading model based on the consortium block chain (DER-CBC) has three vertical and horizontal levels respectively, which is shown in Figure 3. The vertical dimension includes physical structure, transaction process and alliance chain, while the horizontal dimension includes participants, alliance chain and smart contract.

\subsection{Vertical Structure of DER-CBC}

\section{(1) Physical structure model of DER-CBC}

The paper proposes a cross energy distributed energy transaction model based on the alliance chain and whose underlying physical structure is based on the electric energy transaction under the microgrid mode to meet the nearby consumption of distributed power. The model construction is shown in Figure 4.

In the distributed energy trading mode, microgrids are mainly composed of photovoltaic power generation users, wind power generation users, energy storage systems, conventional power generation systems and load. Smallscale photovoltaic and wind power generation users are often the users who combine power generation and consumption. The micro grid energy storage system relates to bus through inverter. Loads mainly include electric vehicles industrial and commercial power, residential power, etc. Some micro

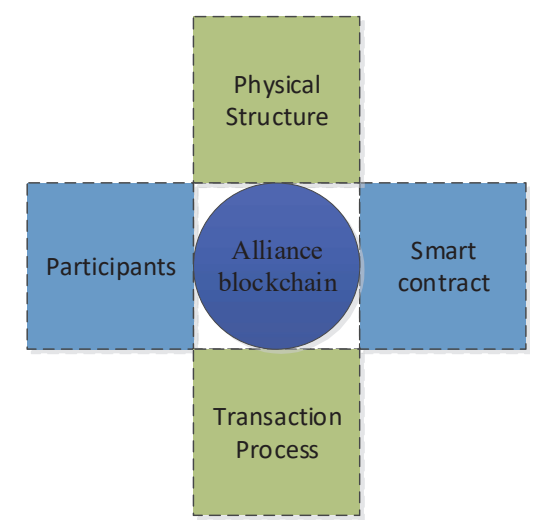

Figure 3 Vertical and horizontal hierarchy of the DER-CBC model. 


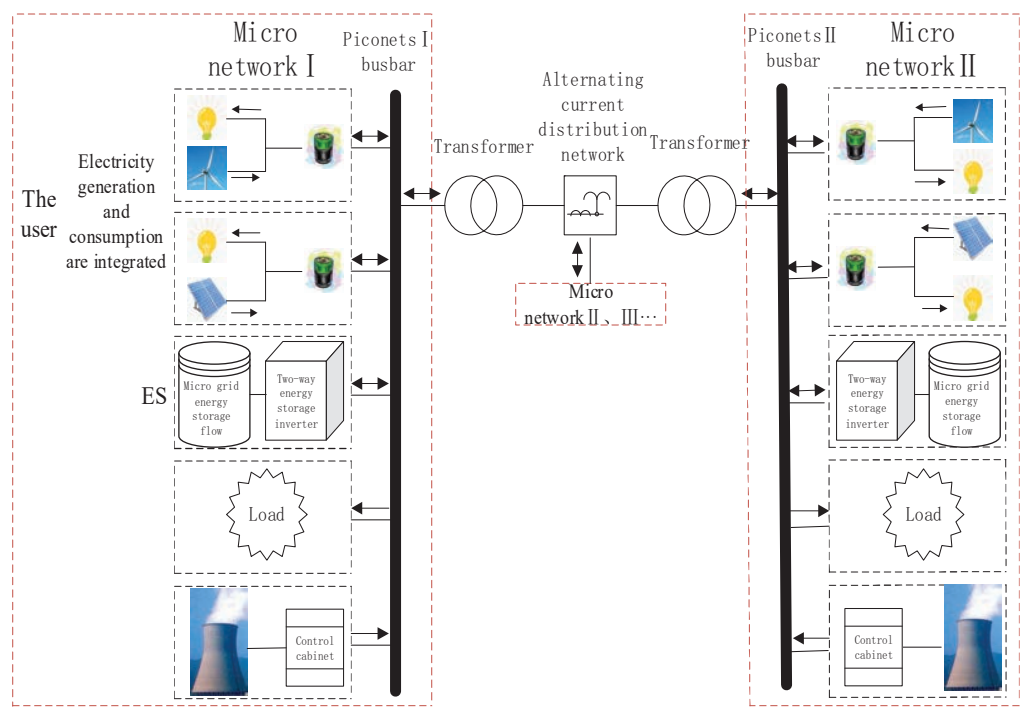

Figure 4 The physical structure of energy trading network.

networks have small power plants. The micro networks are connected through the distribution network.

\section{(2) Transaction process model of DER-CBC}

If the combiner of production and consumption is an electric seller and there is a balance in the electric seller's generation to meet its own demand, the power sale information (ID, power sale, power sale price, geographic information, etc.) can be published on the blockchain power sale platform. If the combiner of production and consumption is an electric buyer, the power demand information (ID, power demand, proposed electricity price, geographic information, etc.) should be released. Then through smart contract matching, the most suitable supplier and demander combination can be food. And then, send the transaction information to suppliers and the demanders. After confirmation, the online contract is signed to clarify electric quantities and prices. Contract parties shall perform according to the contract content. Credit mechanism is introduced to monitor the performance of contracts, including intelligent meter reading, real-time measurement, and electricity pricing. After the above steps are completed, both parties verify and complete the electricity fee settlement. Figure 5 shows the distributed transaction smart contract process. 


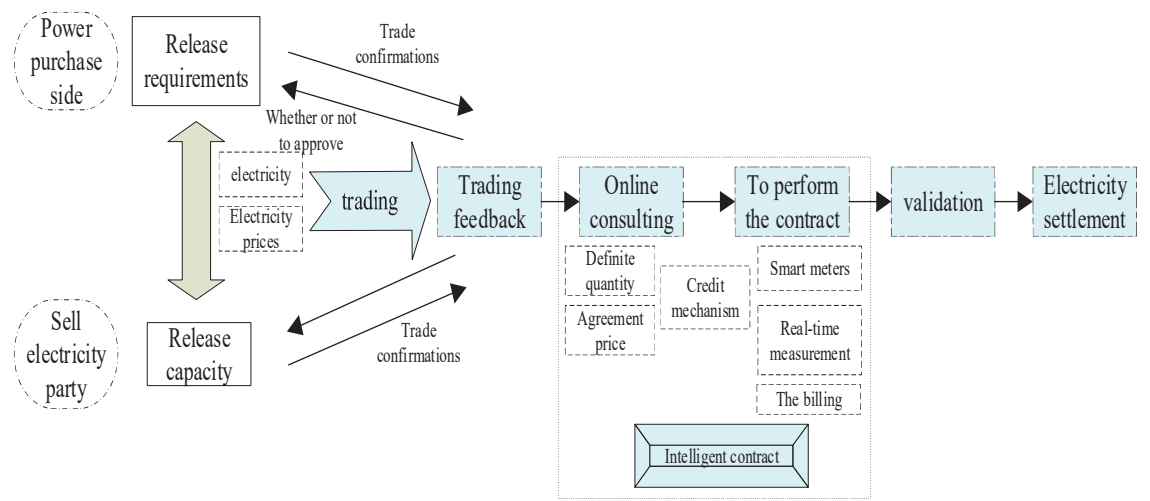

Figure 5 The flow chart of distributed transaction smart contract.

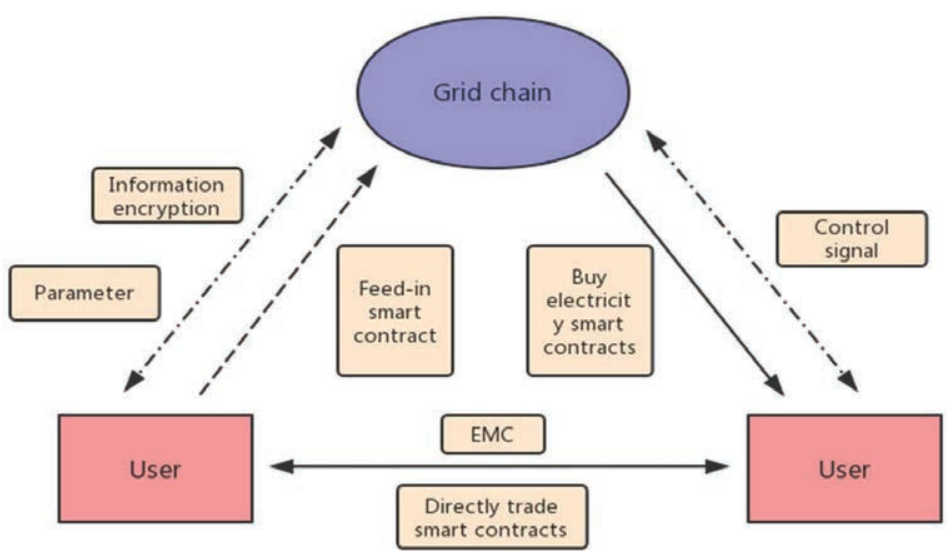

Figure 6 The distributed energy trading model based on consortium block chain.

\subsection{Horizontal Structure of DER-CBC}

The horizontal structure model of DER-CBC includes.

(a) Participants: distributed energy users, power grid companies and regulators in the microgrid.

(b) Blockchain: user chain, power grid chain and energy chain. As shown in Figure 6.

The smart contract containing algorithms can extract information and calculate it automatically. After the calculation, the information will be stored in the blockchain. Transactions are automatically executed and updated in real time. 
(i) User chain recording user information and transaction information, which is shown as formula (1).

$$
D G=\left(I D_{D G}, M_{D G}, D_{D G}, G_{D G}, I_{D G}\right)
$$

Where: $D G$ is the user chain; $I D_{D G}$ is the unique identification obtained after the user joined the der-cbc model and passed the authentication; $M_{D G}$ is the user's power generation capacity; $D_{D G}$ is the user's geographic information; $G_{D G}$ is the user's energy type; $I_{D G}$ is the current user's charging and discharging state.

(ii) The power grid chain information format (2).

$$
P C C(s i)=\left(I D_{P C C}, M_{P C C}, C_{P C C}\right)
$$

Where $P C C$ is the basic information of the public connection point; $s_{i}$ is the node; $I D_{P C C}$ is the unique identity authentication of the public connection point; $M_{P C C}$ is the ability of the public connection point to exchange power with the grid; $C_{P C C}$ is the time-sharing price of the electricity selling company. The supply and demand prices between users should be as close as possible. When there is a new node $s_{i}$ to join, it needs to submit information covering identity ID, power generation capacity, geographic information, and energy type, all of which should get authentication. After the authentication, it will be incorporated into the user chain and participate in the distributed energy transaction.

(iii) The energy chain records basic information about each member and the amount of power they supply. The chain is composed of hydroelectric, thermal, cogeneration, wind farm, photovoltaic, and other power generation companies, and is like the user chain in format (3).

$$
E C=(I D E C, M E C, D E C, G E C)
$$

where: $E C$ is the energy chain; $I D E C$ is the unique identifier obtained by the user after joining the DER-CBC model through authentication; $M E C$ is the member's generation capacity; $D E C$ is the user's geographic information; $G E C$ indicates the user's energy type.

(c) Trading strategy smart contract (TSSC). It consists of three parts: trading strategy smart contract, direct trading smart contract, online smart contract and power purchase smart contract. The work flow of TSSC is shown in Figure 7. 


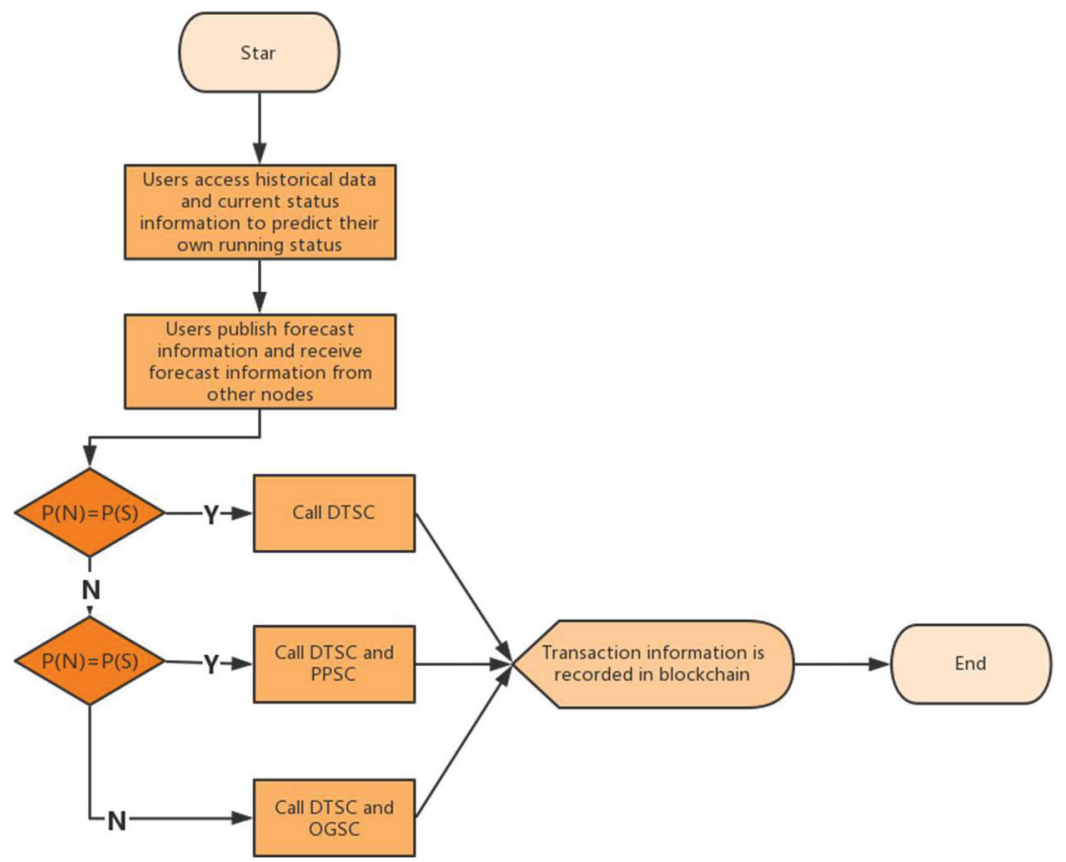

Figure 7 Work flow of TSSC single transaction.

(i) Directly trade smart contract (DTSC)

DTSC is used to define the direct transaction algorithm between users, which meets the following constraint shown in formula (4).

$$
D_{\alpha}=\min \frac{w}{D}
$$

Where $W_{D}$ is the distance weight [Heterogeneous Energy Blockchain for a Multi-Energy Complementary Secure Transaction Model], $D$ is the electrical distance between the power supplier and the power user. According to the notice on launching the pilot market-oriented transaction of distributed generation issued by the National Development and Reform Commission and the National Energy Administration [35], the distributed power is consumed in the local network. When the total installed capacity of distributed generation projects in the network is less than the average power load of the previous year within the power supply scope, the network tariff shall be implemented in accordance with the network tariff standard within the same voltage level. It is approximately assumed that the network tariff is proportional to the 
transmission distance between the power supplier and the power user. The closer the electric distance is, the smaller the $D$ is, the higher the distance coefficient $D \alpha$ is, the higher the priority $w$ is.

Energy supply consensus price chain $\left(I_{a}\right)$ is shown in formula (5).

$$
I_{\alpha}=\min \frac{w}{P_{N}-P_{S}}
$$

Where $w$ is the weight of consensus price difference; $I_{a}$ is the price coefficient of energy supply consensus, which mainly reflects the expected price difference between the supplier and the purchaser in the transaction market. If the power price $P_{s}$ issued by the power supplier is closer to the price $P_{N}$ accepted by the power purchaser, that is to say, the supply and demand prices are as close as possible, the higher the consensus agreement degree of transaction price is, the easier to reach a transaction contract. where $W_{I}$ is the weight of the consensus price difference.

(ii) On-grid smart contract (OGSC)

OGSC is used to define the transaction algorithm for the transaction of surplus electricity in the microgrid, which meets the following constraints shown in formula (6) and formula (7).

$$
G_{\alpha}=\min \frac{w}{Q}
$$

Where $G_{a}$ is the green energy coefficient; $W_{G}$ is the weight of environmental friendliness of the power supplier; $Q$ is the energy cleanliness factor. Setting the cleanliness factor can promote the consumption of new energy such as photovoltaic and wind power, and make new energy have priority to go online. In the paper, set the cleanliness factor of thermal power generation as 0.3 , that of hydropower generation as 0.6 , and that of photovoltaic and wind power generation as 0.8 .

$$
C_{\mathrm{Max}}=\left[\frac{M}{L} \cdot(S G)\right]
$$

Where, $C_{\mathrm{Max}}$ is the decision value to trade energy to the grid, which is a combination of clean environment, capacity value, energy consumption index and other factors; $M$ is the energy produced by a certain power supplier (including each power plant, each distributed capacity unit and individual), and the trading model dispatches each power supply unit according to this capacity value and the power demand side to ensure the stable operation of 
the grid system, and the capacity of the supply side. The larger the energy consumption index, the lower the priority of matching; $L$ is the energy consumption index of a distributed energy source; the larger the energy consumption index, the lower the energy efficiency ratio of the supplier, the less energy saving, and the lower the priority of matching; $S$ is the stability factor of a distributed energy source; the better the quality of power delivered by the supplier, the higher the priority of matching.

(iii) Power purchase smart contract (PPSC)

PPSC is used to define the transaction algorithm of micro grid power purchase from large grid, and record the traditional transaction algorithm with smart contracts. In order to promote the development of distributed energy, higher priority is given to direct transactions between users, and when the supply and demand of electricity between users cannot satisfy each other, the purchase of electricity from power sellers is considered. The economic objectives of distributed trading are achieved on both day-ahead and real-time time scales, including day-ahead and intraday nonlinear economic optimization $[14,15]$. In the day-ahead phase, users upload their own generation or load forecasts to form a day-ahead trading plan. Trading Strategies Smart Contracts (TSSC) determine whether to adopt a strategy of direct trading, feed-in or power purchase, and are stored in the energy chain. In the intraday trading stage, users upload the intra-day ultra-short-term forecast results of their own power generation and load, combine them with the previous day's trading plan, and use the deviation (the difference between the intra-day trading plan and the previous day's trading plan) as an optimization variable to form the final trading plan, which is stored in the energy chain in the form of an alliance chain.

\section{Case Analysis}

\subsection{Design of Algorithms}

The case analysis is based on the blockchain smart contract developed by Ethereum platform, using the Python software to write and deploy smart contract [36-39].

Ethereum is currently one of the mainstream blockchain development platforms. The Ethereum blockchain system is a distributed system with a high degree of data redundancy, consisting of multiple peer nodes (called Ethereum nodes) in Ethereum. The Ethereum blockchain data is the core 
Table 1 Smart contract test scenario settings

\begin{tabular}{lccccc}
\hline Number & $\begin{array}{c}\text { Agreed } \\
\text { Price } \\
(\text { yuan/kWh })\end{array}$ & $\begin{array}{c}\text { Agreed } \\
\text { Volume } \\
(\mathrm{kWh})\end{array}$ & $\begin{array}{c}\text { Agreed } \\
\text { Network } \\
\text { Tariff } \\
(\text { yuan/kWh })\end{array}$ & $\begin{array}{c}\text { Electricity } \\
\text { Consumption of } \\
\text { Customer 1\# }(\mathrm{kWh})\end{array}$ & $\begin{array}{c}\text { Generation of } \\
\text { Generator 2\# } \\
(\mathrm{kWh})\end{array}$ \\
\hline 1 & 0.55 & 200 & 0.02 & 200 & 200 \\
2 & 0.54 & 200 & 0.02 & 220 & 200 \\
3 & 0.56 & 200 & 0.02 & 180 & 200 \\
\hline
\end{tabular}

data in the Ethereum blockchain system, including block data, transaction data, account data, etc. The Ethereum blockchain data is the core data in the Ethereum blockchain system. The Ethereum blockchain data is stored in each Ethereum node and is maintained by all nodes. A device running an Ethereum client program and in the Ethereum blockchain system is an Ethereum node. A user can create a contract by initiating a transaction without a recipient account. The user serializes the contract code (including contract variable definitions, contract function code, etc.) and saves it to the transaction. When the transaction is recorded in the block, the Ethereum smart contract is successfully created and can be invoked by all users [40].

After successful model debugging, it is planned to conduct three smart contract tests first, and then change the parameter to test the DER-CBC model. After successful model debugging, it is planned to conduct three smart contract tests first, then change the parameter and test the model again.

In the case, the sales price is $0.657 \mathrm{yuan} /(\mathrm{kWh})$ with reference to the flat price of $35 \mathrm{kV}$ large industrial users in Beijing; the purchase price of the grid for distributed power generation is set as $0.4 \mathrm{yuan} /(\mathrm{kWh})$; agreed price and agreed network tariff refer to Literature [41]; assume the initial account balance as 100 yuan. In addition, other relevant parameters refer to the corresponding parameter values of Beijing in the third quarter of 2019.

\subsection{Smart Contract Test}

Three accounts are set up to represent electric customer 1\# (power purchaser), distributed energy generator $2 \#$, and electric-selling company respectively. Three smart contract tests are planned to be carried out. The scenario settings are shown in Table 1.

The DER-CBC model test results are shown in Table 2. 
Table 2 Test results of DER-CBC model smart contract

\begin{tabular}{|c|c|c|c|c|c|c|c|}
\hline Number & $\begin{array}{c}\text { Payment } \\
\text { of } \\
\text { Cust- } \\
\text { omer } \\
1 \# \\
\text { (Yuan) }\end{array}$ & $\begin{array}{c}\text { Income } \\
\text { of } \\
\text { Gener- } \\
\text { ator } \\
2 \# \\
\text { (Yuan) }\end{array}$ & $\begin{array}{l}\text { Revenue } \\
\text { of } \\
\text { Electric- } \\
\text { selling } \\
\text { Company } \\
\text { (Yuan) }\end{array}$ & $\begin{array}{c}\text { Account } \\
\text { Balance } \\
\text { of } \\
\text { Customer } \\
1 \# \\
\text { (Yuan) }\end{array}$ & $\begin{array}{c}\text { Account } \\
\text { Balance of } \\
\text { Generator } \\
\text { Customer } \\
2 \# \\
\text { (Yuan) }\end{array}$ & $\begin{array}{l}\text { Account } \\
\text { Balance } \\
\text { of Electric- } \\
\text { selling } \\
\text { Company } \\
\text { (Yuan) }\end{array}$ & $\begin{array}{c}\text { Accumu- } \\
\text { lated } \\
\text { Trading } \\
\text { Volume } \\
\text { (kWh) }\end{array}$ \\
\hline 1 & 114 & 110 & 4 & 86 & 310 & 204 & 200 \\
\hline 2 & 125.14 & 108 & 17.14 & 74.86 & 308 & 217.14 & 420 \\
\hline 3 & 104.4 & 108.8 & -4.4 & 95.6 & 308.8 & 195.6 & 600 \\
\hline
\end{tabular}

In the first test, the electric consumption of customer $1 \#$ is equal to the generation of generator 2\#. Through the judgment of TSSC, DTSC is automatically called to complete the transaction. And the electricity selling company only receives the network tariff.

In the second test, the electric consumption of customer $1 \#$ is more than that of generation of generator 2\#. After judging by TSSC, DTSC and PPSC are automatically called to complete the transaction.

In the third test, the electric consumption of customer $1 \#$ is less than the generation of generator 2\#. After TSSC judgment, DTSC and OGSC are called to complete the transaction.

The results of three tests are consistent with the calculated values of the DER-CBC model, and the DER-CBC model can operate normally.

\subsection{Performance Test of DER-CBC}

\section{(1) User Distance}

In order to test the performance of DER-CBC when its parameters change, the paper simulates and analyses the mining behaviour of market participants on Python platform (it can be understood as the probability of successful transaction [23]). The intelligent contract is used to automatically evaluate users and record them on the blockchain, which affects the mining difficulty of a miner. Set up 10 market entities. Based on Monte Carlo simulation, take the user distance $\mathrm{D}$ as an example to set different parameters. Test the ownership of each block in the mining process of 20 blocks in the distributed energy trading blockchain of the DER-CBC mode, and get the relationship between the user distance $\mathrm{D}$ and the probability of successful mining of the participants in the distributed energy trading. 


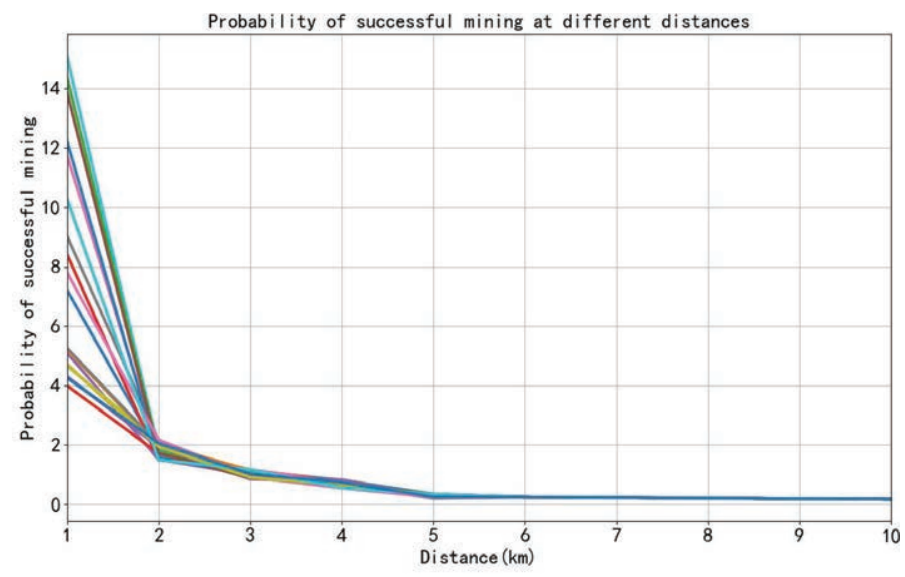

Note: $14 \approx$ Probability $0.8 ; 12 \approx$ Probability $0.7 ; 10 \approx$ Probability $0.6 ; 8 \approx$ Probability 0.5 ; $6 \approx$ Probability $0.4 ; 4 \approx$ Probability 0.3 .

Figure 8 Relationship between successful mining probability of market participants and user distance D.

The vertical axis of Figure 8 represents the probability of successful mining of market participants, and the horizontal axis represents the user distance D. According to the simulation results, successful mining probability of different users is obtained.

According to Figure 8, relevant conclusions can be drawn as followed:

(a) In the DER-CBC model proposed in this paper, the probability of successful mining by market participants (transaction success rate) is positively correlated with the distance of users. The shorter the distance is, the higher the probability of successful mining is.

(b) Under the mechanism set in this paper, the probability of successful mining decreases rapidly with the decrease of user distance. When the user distance reaches a certain value, the probability of successful mining can be almost ignored. That is, when the user distance exceeds a certain value, the direct transaction between users is no longer supported.

\section{(2) Energy Cleanliness}

Similarly, set these 10 market players at the same distance, but with different energy cleanliness, different green energy coefficients $Q$. The simulation results are shown in Figure 9.

The results show that in the DER-CBC model proposed in this paper, the shorter the distance between users and the cleaner the energy is, the higher 


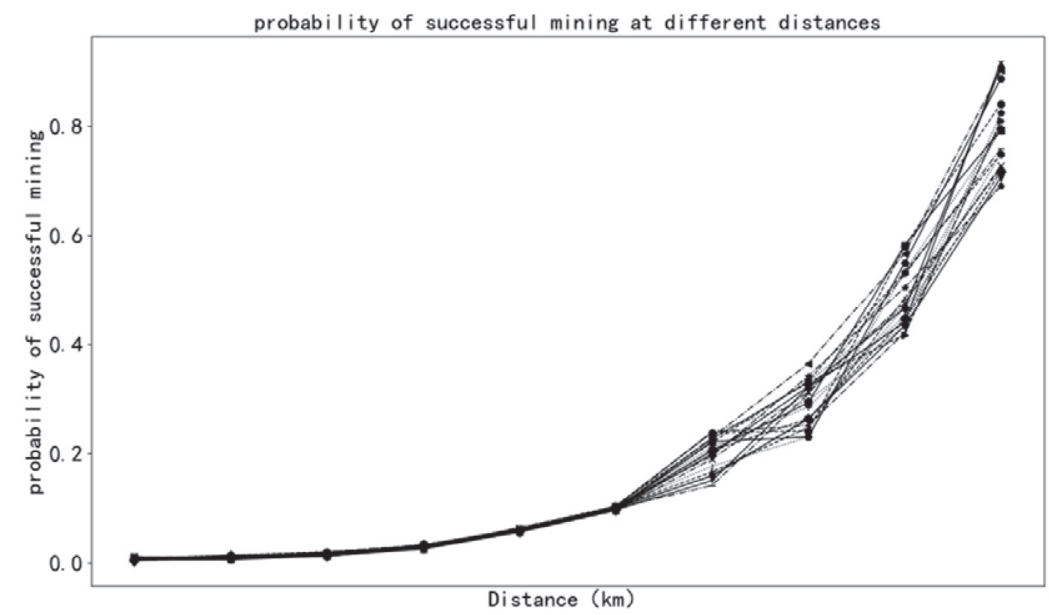

Figure 9 The relationship between the probability of successful mining by market participants and the green energy factor $\mathrm{Q}$.

the probability of mining success, thus providing an incentive for more economical distributed energy trading, encouraging clean energy consumption, and increasing distributed energy usage. The constraints of DER-CBC mainly consider the optimization of user distance, power supply intermittence and green energy utilization rate. Other factors can be added according to the actual situation.

\section{Conclusions}

The decentralized, intelligent, open, and transparent features of blockchain are in line with the trend of energy development, providing technical support for the development of the energy industry and pointing to a new direction for future development in distributed energy network transactions [42]. Distributed energy transactions based on the Alliance blockchain are not only conducive to transparent and convenient distributed energy network transactions, but also ensure effective centralized control and optimization of the distributed energy trading platform.

According to the development of distributed energy in China and the characteristics of blockchain technology, this paper constructs a distributed energy transaction model based on alliance blockchain (DER-CBC), which involves two dimensions: the vertical dimension includes physical structure, transaction process and alliance chain, while the horizontal dimension 
includes participants, alliance chain and smart contract. The paper uses Python to build and simulate the DER-CBC model on the Ethereum platform, where the smart contract running status and the user behaviour in the model when parameters change is tested. The paper aims to provide a new idea for building a distributed trading platform and promoting the development of distributed energy by using blockchain technology. The future distributed energy trading alliance blockchain market may be in the form of multichain collaboration, cross-chain circulation of source network load storage, Internet of Things, micro-grid, power trading and other areas through the interconnection of different blockchains using multi-chain forms to solve energy trading problems [43], forming a "small physical network + large information network + side chain assistance". The pattern of the blockchain includes energy producers and consumers, energy companies and government regulators and other relevant individuals, groups, and institutions under the framework of the energy alliance chain. In the long run, the combination of blockchain and the energy industry is the general trend of the energy industry [44].

Alliance blockchain plays a positive role in the energy sector, but blockchain technology, as a nascent technology, still needs to pay attention to its risks when applied, [45] first of all, on the issue of information security and optimization of data processing on the regional chain, blockchain technology allows nodes in the block to add custom information and has the characteristic of being immutable after determination, which improves its security, optimization, and security of the block. Data processing steps, but if there are hackers in the custom information to add cracked information, the whole alliance blockchain will be at great risk. Secondly, with the continuous changes in national electricity demand, the energy system is becoming richer and richer in all kinds of subjects, and the energy transaction methods are more diversified, while the existing technology may be unstable in the operation of the whole system in the alliance blockchain [46].

The advantages of blockchain are not just in the technology itself, but in the use of the technology to embody organizational advantages and to create a system that is jointly maintained by multiple market participants. In order to better promote the application of blockchain technology in the energy industry, it must be constrained by institutions and policies. The state controls and regulates the energy industry, and energy trading belongs to the field of state control, so the combination of alliance blockchain and energy and electricity should be carried out based on policy permission, and the application of alliance blockchain in distributed energy trading needs the 
support of relevant policies and regulations [18]. The future development of alliance blockchain energy trading needs to find development solutions that are both suitable for the energy trading market and keep up with policy requirements, and relevant state departments should improve existing policies and regulatory systems to maximize support for alliance blockchain technology development [47]. Take a holistic perspective on all aspects of the energy blockchain construction, various levels and elements of overall consideration, strengthen the Alliance blockchain top-level design and overall planning, accelerate the Alliance blockchain and cloud, large, material, migration, wisdom and other technologies of deep integration, promote integration and innovation and integration of applications, internal quality and efficiency, external integration and development [48].

In this paper, although the advantages of blockchain in data storage and security and mutual trust are utilized in energy transactions to achieve direct transactions between the supply and demand sides of energy, this paper is still in the exploratory stage of how to adjust the rules of the transaction according to the actual energy supply and demand relationship in the region to maximize the development and consumption of clean energy.

\section{Acknowledgment}

The paper is supported by the Fundamental Research Funds for the Central Universities (Grant No. 2652019083), the Beijing Municipal Social Science Foundation (17YJC029), the Technology Innovation Project of Changed Economic Research Institute in 2020 (Integration of Blockchain and Energy Internet: a Case of Clean Energy Distributed Transaction Model), the National Natural Science Foundation of China (Grant No. 51978443), and the Central University Basic Scientific Research Business Expenses Special Funds (Project No: 2017XS096).

\section{References}

[1] Felstead ØD, Snow CC. Business models and organization design. Long Range Planning 2017. Available online.

[2] Zeng Ming, Yang Yangqin, Liu Duncan, et al. Coordination and optimization of operation mode and key technologies of energy Internet "sources-nett-hold-storage" [J]. Power grid technology, 2016, 40(1):114-124. 
[3] Energy Network - China Energy News. It is very difficult for distributed energy generation to access the Internet [EB/OL]. Https://www.in-en.co $\mathrm{m} /$ article/html/energy-2222426.shtml

[4] Rifkin J. The third industrial revolution: how lateral power is transforming energy, the economy, and the world [M]. New York: Palgrave MacMillan, 2011.

[5] Yi Wang, Ning Zhang, Chongqing Kang. Research review and prospect on optimization planning and operation of energy hubs in energy Internet [J]. Chinese journal of electrical engineering, 2015, 35(22):56695681.

[6] Su W, Huang AQ. A game theoretic framework for a next-generation retail electricity market with high penetration of distributed residential electricity suppliers [J]. Applied Energy, 2014, 119(C):341-350.

[7] Zhou K, Yang S, Zhen S. Energy Internet: The business perspective [J]. Applied Energy, 2016, 178:212-222.

[8] Lei Tan, Gang Chen. Block chain 2.0 [M]. Beijing: electronic industry press, 2015.

[9] Ning Zhang, Yi Wang, Chongqing Kang, Jian nan Cheng, He Dawei. Block chain technology in energy Internet: research framework and typical applications [J]. Chinese journal of electrical engineering, 2016, 36(15):4011-4023.

[10] Jing Lu, Bin Song, Wanhong Xiang, Zhiming Zhou. Intelligent contract for power market transaction settlement based on block chain [J]. Computer system application, 2017, 26(12):43-50.

[11] Ding Wei. Block chain-based instrument data management system. China Instrumentation, 2015, (10):15-17.

[12] Zhigang Jin, Ruoxi Wu, Gen Li, Yue Shunmin. Ev charging transaction model based on the soyuz block chain [J]. Power grid technology, 2019, 43(12):4362-4368.

[13] Hongling Xie, Yanan Zheng, Yanqing Li. Microgrid economic transaction model based on energy block chain network [J]. Journal of north China electric power university, 2019, 46(3):17-26.

[14] Yanan Zheng. Microgrid scheduling and trading method based on energy block chain [D]. North China electric power university, 2019.

[15] Wei She, Zhihao Gu, Xiaoyu Yang, Zhao Tian, Jiansen Chen, Wei Liu. Multi-energy complementary security trading model of heterogeneous energy block chain [J]. Power grid technology, 2019, 43(9):3193-3201. 
[16] Cai Weide, Yu Lian, Wang Rong, et al. Research on application development method based on blockchain [J]. Journal of Software, 2017(6):1474-1487.

[17] Wey, A.B., et al. Public chain technology and its application value [J]. Internet Economy, 2018(07):26-31.

[18] Wei Bin, Liu Xiaofeng, Gou Hang. An investigation of the distributed on-chain energy transaction model based on public chain [J]. Journal of Changchun Normal University, 2020, 39(02):41-47.

[19] Kazi Masudul Alam, J.M. Ashfiqur Rahman, Anisha Tasnim, Aysha Akther, A Blockchain-based Land Title Management System for Bangladesh, Journal of King Saud University - Computer and Information Sciences, 2020.

[20] Li Zhibin. Research on Access Control of Healthcare Security Network Based on Ethereum Private Chain [D]. Huazhong University of Science and Technology, 2019.

[21] Rui Hou, Guowen Ren, Chunlei Zhou, et al. Analysis and research on network security and privacy security in ubiquitous A Certificate Storage Method for Confirming Video Copyright and Tracing the Source Based on Electricity Internet of Things. 2020, 158:64-72.

[22] Yang Yang, Yang Yang, Yu Dingguo. A Certificate Storage Method for Confirming Video Copyright and Tracing the Source Based on Alliance Chain. 2020, 1631(1):012180.

[23] Zhang Lihua, Hu Fangzhou, Huang Yang, Wan Yuanhua, Li Jingjing. A microgrid identity protocol based on federated chains [J]. Journal of Applied Science, 2020, 38(01):173-183.

[24] Qiao Rui, Cao Yan, Wang Qingxian. A dynamic data traceability mechanism for the Internet of Things based on federated chains [J]. Journal of Software, 2019, 30(06):1614-1631.

[25] XIA Chenyi, CAI Qingsong, WU Jie. A quoteless trading mechanism for microgrid based on PoA alliance chain[J]. Computer System Applications, 2020, 29(11):57-65.

[26] Cheng Dongling, Shen Chen, Pang Ling. Research on the Application of Alliance Blockchain in Energy Internet [J]. Mechatronics Information, 2019(24):155-157.

[27] Yang Dechang, Zhao Xiaoyu, Xu Zixiang, Li Yong, Li Qiang. Analysis of the current situation and prospect of blockchain application in energy internet $[\mathrm{J}]$. Chinese Journal of Electrical Engineering, 2017, 37(13):3664-3671. 
[28] Zhengtang Fu, Peiwu Dong, Yanbing Ju, an intelligent electric vehicle charging system for new energy companies based on consortium blockchain, Journal of Cleaner Production, Volume 261, 2020, 121219.

[29] Shaomin Zhang, Jieqi Rong, Baoyi Wang, A privacy protection scheme of smart meter for decentralized smart home environment based on consortium blockchain, International Journal of Electrical Power \& Energy Systems, Volume 121, 2020, 106140.

[30] Qiang Wang, Min Su, Integrating blockchain technology into the energy sector -from theory of blockchain to research and application of energy blockchain, Computer Science Review, Volume 37, 2020, 100275.

[31] Yi Zhang, Qian Shi, an intelligent transaction model for energy blockchain based on diversity of subjects, Alexandria Engineering Journal, 2020.

[32] Jianchao Hou, Che Wang, Sai Luo, how to improve the competiveness of distributed energy resources in China with blockchain technology, Technological Forecasting and Social Change, Volume 151, 2020, 119744.

[33] Xin Zhou, Lirong Deng, Bin Wang, Zhaoguang Pan. Decentralized energy trading system based on alliance chain [J]. Global energy Internet, 2019, 2(6):556-565.

[34] Lihua Zhang, Fangzhou Hu, Yang Huang, Yuanhua Wan, Jingjing Li. Micro grid identity authentication protocol based on alliance chain [J]. Journal of Applied Sciences, 2020, 38(1):173-182.

[35] National Energy Administration of the national development and Reform Commission. Notice on carrying out the pilot market-oriented transaction of distributed generation [EB/OL]. Http://zfxxgk.nea.gov.c n/auto87/201711/t2017113.htm.

[36] Python and flask framework development Ethereum smart contract [DB/OL]. https://segmentfault.com/a/1190000016188023.

[37] Python interacts with Ethereum [DB/OL]. https://blog.csdn.net/g84333 73/article/details/82432766.

[38] Ethereum Python smart contract development guide [DB/OL]. https: //blog.csdn.net/mongo_node/article/details/85043799.

[39] Wes Mckinney. Use Python for data analysis [M]. Mechanical industry press, 2019: 361-424.

[40] Xiaoju Huang. Research and implementation of Geth-EBTree, an Ethereum blockchain data query system [D]. East China Normal University, 2020. 
[41] Ping Jian, Yan Zheng, Chen Sijie, Shen Zeyu, Yang Su, Li Jing, Qu Haoyuan. Credit risk management method of distributed energy trading market based on block chain [J]. Chinese journal of electrical engineering, 2019, 39(24):7138-7145.

[42] Adetomike Adeyemi, Mingyu Yan, Mohammad Shahidehpour, Cristina Botero, Alba Valbuena Guerra, Niroj Gurung, Liuxi (Calvin) Zhang, Aleksi Paaso, Blockchain technology applications in power distribution systems, The Electricity Journal, Volume 33, Issue 8, 2020, 106817.

[43] Li Bin, Qin Qiu Yue, Qi Bing, Sun Yi, Li De-zhi, Shi Kun, Yang Bin, Xi Peifeng. An overview of blockchain-based distributed energy trading scheme design [J]. Power Grid Technology, 2019, 43(03):961-972.

[44] Shang Helm. Application prospect of blockchain technology in energy industry[J]. Power Information and Communication Technology, 2019, 17(02):1-8.

[45] Biryukov A, Khovratovich D, Pustogarov I. Deanonymisation of clients in Bitcoin P2P network [C]. Proceedings of the 2014 ACM SIGSAC Conference on Computer and Communications Security, 2014:15-29.

[46] Yu Xiaobao, Zheng Dan dan. Application and Prospect of Blockchain Technology in the Energy and Power Sector [J]. Huadian Technology, 2020, 42(08):17-23.

[47] Shuai Zhu, Malin Song, Ming Kim Lim, Jianlin Wang, Jiajia Zhao, The development of energy blockchain and its implications for China's energy sector, Resources Policy, Volume 66, 2020, 101595.

[48] Yang Ke, Xuan Jiaxing, Wang Huanjuan, Li Guomin, Shen Xueqing. A Review of Blockchain Technology in Energy and Power Industry [J]. Power Construction, 2020, 41(11):1-15. 


\section{Biographies}

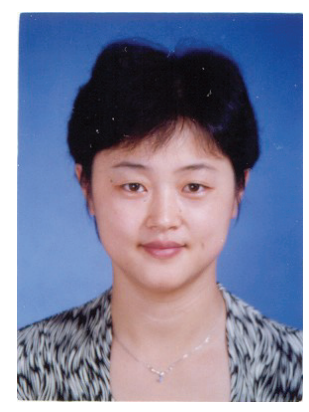

Yongxiu He graduated from north China electric power university in 1997, had a master's degree in power system and its automation major, received a doctor's degree in management of north China electric power university, 2006, April 2004 - December 2009, for north China electric power university institute of business administration power economic management department, responsible for the direction of the power of economic and management specialized teaching and scientific research management. Since December 2009, she has been the Deputy Dean of School of Economics and Management of North China Electric Power University, responsible for teaching management of School of Economics and Management of North China Electric Power University. In the electric power technology economy and management, electricity market, energy economy, low carbon economy and other fields of in-depth research.

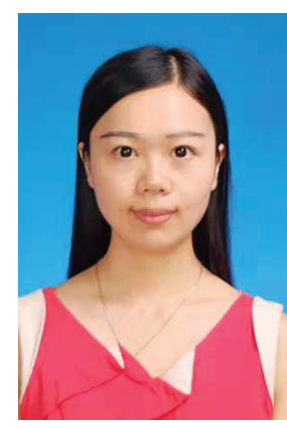

Wei Xiong is a Ph.D. student in Energy Management of North China Electric Power University, engaged in research on the application of blockchain technology in the energy industry. 


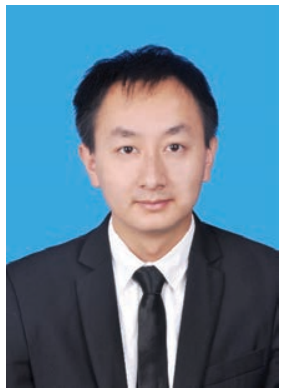

Bin-you Yang graduated from Changsha University of Science and Technology in 2019 with a master's degree in electrical engineering. After graduation, he worked in State Grid Changde Power Supply Company and is now engaged in research work on power market economy.

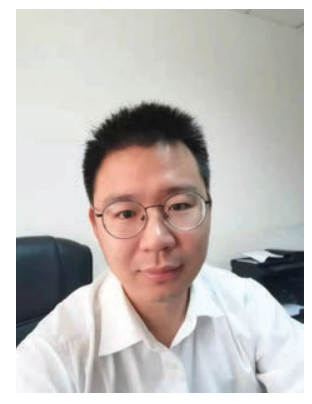

Rui Zhang graduated from Guangdong University of Technology in 2014 with a master's degree in electrical engineering. After graduation, he worked in State Grid Changde Power Supply Company. Now he is the director of distribution department in Hanshou Power Supply Company of State Grid, engaged in electric power big data analysis and other research work. 


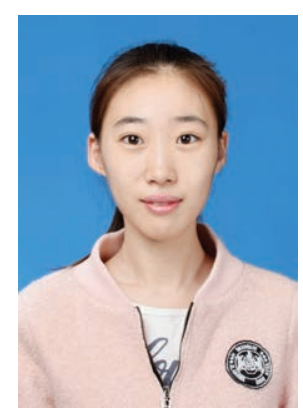

Ming-li Cui graduated from the School of Economics and Business Administration of Heilongjiang University in 2021. Ms Cui has won many honors such as national scholarship, academic scholarship and study model during her college years. In 2020, she was admitted to China University of Geosciences (Beijing) as the first student in her major and enrolled in September 2021. At present, Ming-li Cui has joined her tutor's research group and actively completed the project research.

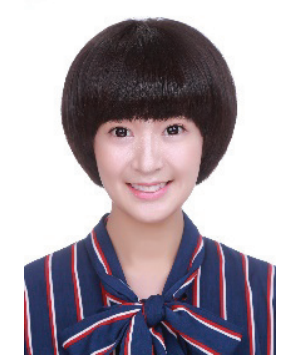

Tian-tian Feng, Doctor of Technology Economics and Management, School of Economics and Management, China University of Geosciences, Beijing, China. Tian-tian Feng was born in Henan province, china, in 1989. She received her master degree and Ph.D degree of technology economics and management from North China Electric Power University in 2013 and 2016. She is currently working in School of Economics and Management, China University of Geosciences. Her research mainly concerns on renewable energy, electricity economics and energy economics. 


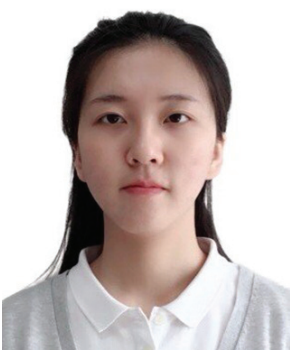

Yi-er Sun is a M.Sc student in Mechanical Engineering at The Hong Kong University of Science and Technology. Her main research direction is environmentally friendly intelligent building robots. She has been involved in the learning of LCA and green management, and the improvement of China's CLCD database. She attended China University of Geosciences (Beijing) in 2016, where she received a B.Sc in ME and a double degree in Business Administration. She is very interested in interdisciplinary field such as energy economy and industrial development. She participated in the research of blockchain application in the green certificate during her undergraduate period, as well as the discussion of the green certificate mechanism. 
